\title{
MODELING OF WAVE-INDUCED SEDIMENT TRANSPORT AROUND OBSTACLES $^{1}$
}

\author{
Stéphan Grilli² ${ }^{2}$ Jeffrey Harris ${ }^{2}$, and Nathanael Greene ${ }^{2}$
}

\begin{abstract}
We report on further developments of a hybrid numerical model to simulate wave-induced sediment transport. A 2D numerical wavetank (NWT) based on fully nonlinear potential flow (FNPF) equations is used to simulate fully nonlinear wave generation and propagation. A 3D Navier-Stokes model with large eddy simulation (LES) is coupled to the NWT to simulate complex turbulent flows near the ocean bottom or around obstacles. Wave kinematics in the 2D-NWT thus forces flow simulations in the 3D-NS-LES model, and resulting sediment transport over the seabed and around a partially buried obstacle. The latter is calculated in a non-cohesive suspended load transport model simulating the (scalar) sediment concentration, using a constant settling velocity. The 2D NWT is based on a higher-order boundary element method (BEM), with explicit 2nd-order time stepping. The computational grid, thus, is the 2D-NWT boundary and the 3D-LES near-field domain. In the present new formulation, the total velocity and pressure fields are expressed as the sum of irrotational (incident/far-field) and near-field viscous perturbations. The LES equations are formulated and solved for the perturbation fields only, which are forced by the incident fields computed in the NWT. The feasibility of coupling the models in an efficient manner is demonstrated.
\end{abstract}

\section{INTRODUCTION}

Despite the vast increase in performance of modern computer clusters, in numerical simulations of complex flow problems, for optimum efficiency, one typically limits the numerical model "physics" (i.e., such as represented in its equations and boundary conditions) to that necessary and sufficient for solving a given problem. For problems with multiple scales or large inhomogeneities in model domain geometry and/or nature, the minimum required "physics" might turn out to be different for different parts of the computational domain (e.g., the wave shoaling zone and the surfzone). Hence, one is faced with either using the more accurate physics everywhere (a costly choice) or adapting it to the nature of the problem, in various parts of the computational domain. The latter is best achieved through model coupling or, even better, through using a hybrid model, in which say two different models' equations and numerical procedures are fully integrated with each other and, if necessary, feedback from the second model onto the first one may be simulated.

Coupled/hybrid models have been used for ocean wave simulations. Once generated, waves closely behave as irrotational inviscid flows, well simulated by potential flow theory up to overturning (New et al. 1985; Grilli 1997; Dommermuth et al. 1998). [Note, when using fully nonlinear free surface boundary

\footnotetext{
${ }^{1}$ To appear in Proc. 32nd Intl. Coastal Engng. Conf. (Hamburg, Germany, September, 2008). ASCE Publications.

${ }^{2}$ Department of Ocean Engineering, University of Rhode Island, Narragansett, RI 02882, USA; grilli@oce.uri.edu
} 
conditions, one refers to these models as "Fully Nonlinear Potential Flow" models (FNPF).] Interactions of waves with ocean structures or the beach, however, cause flow separation and/or breaking, requiring the use of viscous/turbulent models, with a free surface description that allows for the representation of breaking waves (as in a Volume Of Fluids (VOF) approach; e.g., Lin and Liu 1998; Lubin 2004). Such models typically solve 3D Navier-Stokes (NS) equations over a fine grid, and are thus very computationally intensive. They may also suffer from excessive numerical dissipation over long distances of propagation, implying that incident gravity waves would dissipate their energy in an excessive manner when propagated in large domains. For this reason, coupled (or hybrid) FNPF-NS-VOF models were developed in 2D and 3D to study wave shoaling and breaking on slopes and beaches, and wave-structure interactions (e.g., Biausser et al. 2004; Helluy et al. 2005; Corte and Grilli 2006). In these problems, incident waves in the FNPF model were either 2D FNPF solitary waves, wavemaker generated long waves (Grilli 1997), or 3D extreme periodic waves (a.k.a., freak waves) generated through directional focusing (Fochesato et al. 2007).

Gilbert et al. (2007) similarly developed a 2D-FNPF/3D-NS hybrid model to study wave-induced sediment transport and scouring around bottom obstacles (Fig. 1). "Far-field" waves are generated in the 2D-FNPF model (by a wavemaker on $\Gamma_{w}$; Grilli and Subramanya 1996 (GS); Grilli and Horrillo 1997 (GH); Grilli et al. $2003(\mathrm{GE})$ ) and propagated over a sloping bottom, up to close to breaking (before dissipation in an absorbing beach; AB). Energy dissipation due to bottom friction or breaking is specified over the (sandy) slope (for $x \leq x_{r}$ ) and in the absorbing beach for $x \geq x_{a}$. A small partly-buried cylinder is located on the bottom, just before the surfzone. The FNPF model thus computes forcing wave fields around the bottom obstacle, that are used to force a coupled 3D-NS model, with embedded sediment transport computation, applied in a "Near-field" computational domain specified around the obstacle.

In this paper, we report on both recent improvements and validations of this hybrid model for wave-induced sediment transport. In the present formulation, the total velocity and pressure fields are expressed as the sum of irrotational (incident/far-field) and near-field viscous perturbations. The NS equations are formulated and solved for the perturbation fields only, which are forced by the incident fields computed in the NWT. The feasibility of coupling the models in an accurate and efficient manner is demonstrated in applications.

\section{METHODOLOGY}

We briefly present equations for the coupled hybrid model, whereby the irrotational wave flow in a 2D-FNPF Numerical Wave Tank (NWT) forces computations in the 3D-NS model with Large Eddy Simulations (LES).

\section{D Numerical Wavetank}

For FNPF flows with a free-surface, we define $\Phi(x, y, z, t)$, the velocity potential, such that velocity, $u_{i}^{I}=\partial \Phi / \partial x_{i}$ satisfies Euler's equations. Thus, mass 


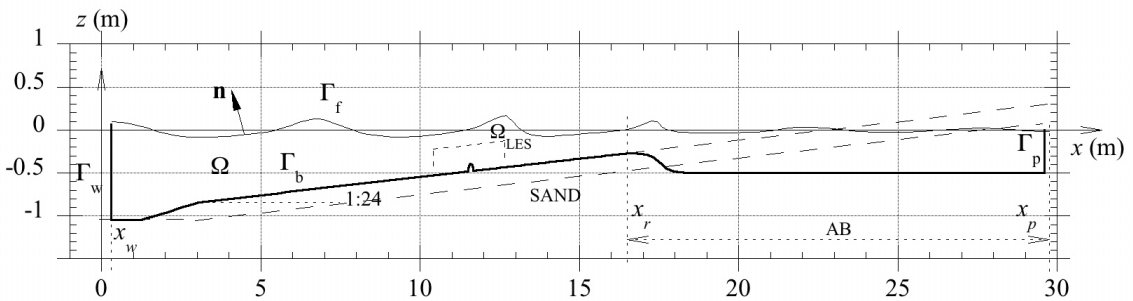

Figure 1: Sketch of 2D-FNPF simulations for wave-induced sediment transport around a bottom obstacle. The 3D-NS-LES domain is marked as $\Omega_{L E S}$.

conservation becomes Laplace's equation (left), which is efficiently solved using a Boundary Element Method (BEM) based on Green's 2nd identity (right),

$$
\nabla^{2} \Phi=0 \quad ; \quad \alpha \Phi\left(x_{i}\right)=\int_{\Gamma}\left\{\frac{\partial \Phi}{\partial n} G\left(x, x_{i}\right)-\Phi \frac{\partial G\left(x, x_{i}\right)}{\partial n}\right\} d \Gamma
$$

where $\Gamma$ denotes the NWT boundary (Fig. 1), $n$ is the outwards normal vector to the boundary, and the 2D free-space Green's function reads, $G\left(x, x_{l}\right)=$ $-(1 / 2 \pi) \ln \left|x-x_{l}\right|$, with $x_{l}$ a collocation node on the boundary. Fully nonlinear kinematic and dynamic boundary conditions are specified on the free-surface $\Gamma_{f}$ (in a mixed Eulerian-Lagrangian formulation (MELF)),

$$
\frac{D r_{i}}{D t}=\frac{\partial \Phi}{\partial x_{i}} \quad ; \quad \frac{D \Phi}{D t}=-g x_{3}+\frac{1}{2} \frac{\partial \Phi}{\partial x_{i}} \frac{\partial \Phi}{\partial x_{i}}-\frac{p_{a}}{\rho}
$$

respectively, where $x_{3}$ is the vertical coordinate, and a (Neumann) no-flow condition is specified on the other boundaries, whether a moving wavemaker $\left(\Gamma_{w}\right)$ or lateral absorbing boundary $\left(\Gamma_{p}\right)$, and the seabed $\left(\Gamma_{b}\right)$. The free surface potential and geometry $\left(r_{i}\right)$ are then time integrated, using 2nd-order Taylor series expansions in a MELF (i.e., applied to both $\Phi$ and $\partial \Phi / \partial t$ ), in which Eqs. (2) and their time derivative, provide 1st and 2nd-order terms respectively.

For the 2D-NWT applications shown in this manuscript, including determining the solution at internal points, details of numerical methods can be found in Refs. (GS,GH,GE).

\section{D-NS with Large Eddy Simulations}

NS equations for an incompressible, isothermal, Newtonian fluid read,

$$
\frac{\partial u_{i}}{\partial x_{i}}=0 \quad ; \quad \frac{\partial u_{i}}{\partial t}+\frac{\partial}{\partial x_{j}}\left(u_{i} u_{j}+\frac{p}{\rho} \delta_{i j}-\nu \frac{\partial u_{i}}{\partial x_{j}}\right)=0
$$

where $u_{i}$ and $p$ are velocity and dynamic pressure, respectively, in a fluid of density $\rho$ and kinematic viscosity $\nu$. We then employ a decomposition of the flow into a free-stream (incident wave) velocity $u_{i}^{I}$ and pressure $p^{I}$ and a perturbation velocity $u_{i}^{P}$ and pressure $p^{P}$,

$$
u_{i}=u_{i}^{I}+u_{i}^{P} \quad ; \quad p=p^{I}+p^{P} .
$$


The incident wave flow $u_{i}^{I}$ computed in the 2D-NWT is assumed inviscid and irrotational, and hence satisfies Euler equations,

$$
\frac{\partial u_{i}^{I}}{\partial x_{i}}=0 \quad ; \quad \frac{\partial u_{i}^{I}}{\partial t}+\frac{\partial}{\partial x_{j}}\left(u_{i}^{I} u_{j}^{I}+\frac{p^{I}}{\rho} \delta_{i j}\right)=0
$$

[Which is quite accurate in nature, outside of a thin oscillatory bottom boundary layer.] After some simplications, we find equations for the perturbation fields,

$$
\frac{\partial u_{i}^{P}}{\partial x_{i}}=0 \quad ; \quad \frac{\partial u_{i}^{P}}{\partial t}+\frac{\partial}{\partial x_{j}}\left(u_{i} u_{j}-u_{i}^{I} u_{j}^{I}+\frac{p^{P}}{\rho} \delta_{i j}-\nu \frac{\partial u_{i}}{\partial x_{j}}\right)=0
$$

By applying a filter (denoted by an overbar) to NS equations we obtain a momentum equation for the resolved perturbation flow,

$$
\frac{\partial \bar{u}_{i}^{P}}{\partial x_{i}}=0 \quad ; \quad \frac{\partial \bar{u}_{i}^{P}}{\partial t}+\frac{\partial}{\partial x_{j}}\left(\bar{u}_{i} \bar{u}_{j}-\overline{u_{i}^{I} u_{j}^{I}}+\frac{\overline{p^{P}}}{\rho} \delta_{i j}-\nu \frac{\partial \bar{u}_{i}}{\partial x_{j}}+\tau_{i j}\right)=0 \quad \text { (7) }
$$

where the subgrid scale (SGS) stress is defined as, $\tau_{i j}=\overline{u_{i} u_{j}}-\bar{u}_{i} \bar{u}_{j}$ and modeled in the LES with a Smagorinsky scheme.

The resulting equations are discretized similar to Zang et al. (1994) and Cui and Street (2001), i.e., using 2nd-order methods in both time and space. QUICK (Leonard 1979) is used to discretize the convective terms of the fluid flow, and 2nd-order differences are used for the remaining terms. The convective terms are integrated in time using the 2nd-order Adams-Bashforth technique, and the diffusive terms are treated with an 2nd-order implicit Crank-Nicolson scheme. The Poisson equation for the pressure field is solved with a multigrid technique. Note, in our hybrid approach, the 2D-FNPF BEM model computes the $I$ fields at the NS mode grid points, directly and explicitly from the boundary solution at time $t$, without any additional approximations.

\section{Non-cohesive Sediment Transport Model}

In work in progress, the strongly coupled hybrid model (2D-NWT and 3DNS-LES) is used to simulate non-cohesive sediment transport and resulting scouring around a partially buried rigid object on the bottom (Gilbert et al. 2007), induced by fully nonlinear irregular (Greene 2008) shoaling waves (Figs. 1). Thus, (scalar) suspended sediment concentration $C$ i(by volume fraction) is modeled with a volume-filtered advection-diffusion equation,

$$
\frac{\partial \bar{C}}{\partial t}+\frac{\partial}{\partial x_{j}}\left\{\left(u_{j}^{I}+u_{j}^{P}-w_{s} \delta_{i 2}\right) \bar{C}+\overline{u_{j} C}\right\}=0
$$

where $w_{s}$ is sediment fall velocity (function of sediment properties, size and shape). Consistent with the common assumption that the turbulent Schmidt number is near unity, the same Smagorinsky SGS turbulence model is adopted for the fluctuation terms in both the momentum and sediment concentration $\left(\overline{u_{j} C}\right)$ equations. SHARP (Leonard 1988) is used to discretize the advective terms of the suspended sediment concentration. 


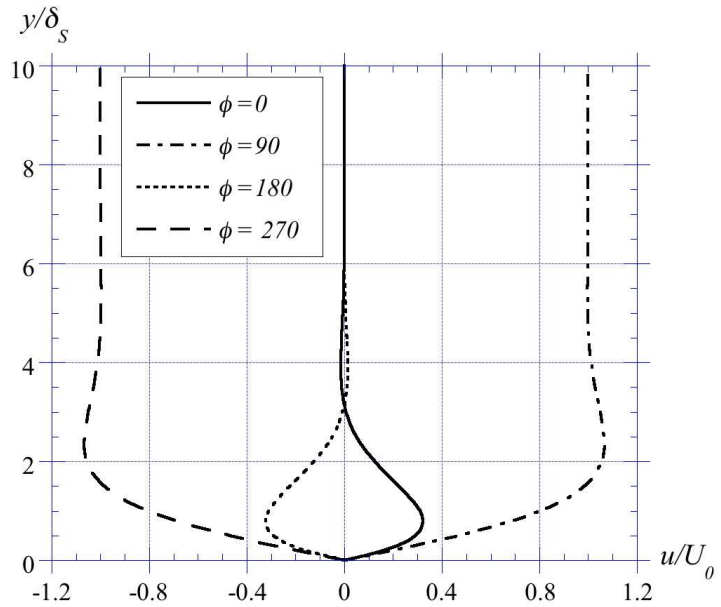

Figure 2: Laminar BL solution (Eq. 9), for phase angle $\phi=\omega t$ (deg.).

\section{APPLICATIONS}

We present here recent 2D-NWT/3D-LES hybrid model simulations, formulated for the perturbation fields as detailed above, namely cases of wave-induced flows near a smooth or rough bottom, and sediment transport induced around a partly buried cylinder.

\section{Oscillatory Laminar Boundary Layer}

The idealized Stokes problem is first used to investigate the hybrid model's accuracy and convergence. This represents an oscillatory flow over a smooth (infinite) solid plate, for which there is an analytic solution. The irrotational flow forcing the NS model, here, is simply a specified uniform harmonic current $U^{I}(y, t)=U_{o} \sin \omega t$, of period $T=2 \pi / \omega$, in the $x$ direction, with $y$ the vertical direction; hence, here there is no need for computations in the NWT.

Spatial periodicity of the NS solution for the $P$ fields is specified in the $x$ and $z$ directions. A no slip condition $u_{i}=0$ is specified on the bottom boundary at $y=0$, and the free stream velocity $U^{I}$ is specified at $y=h$ above the plate, with $(v=w=0 ; \partial u / \partial y=0)$. For a low Reynolds number Re, the $($ mean $=$ total $)$ velocity $u(y)=U=U^{I}+U^{P}$ near the plate takes the form of an oscillatory laminar boundary layer expressed by,

$$
u(y)=U_{0}\left\{\sin \omega t-\exp \left(\frac{-y}{\delta_{S}}\right) \sin \left(\omega t-\frac{-y}{\delta_{S}}\right)\right\}
$$

with $\delta_{S}=\sqrt{2 \nu / \omega}$, the Stokes layer thickness. This solution applies for $\operatorname{Re}=$ $U_{o} \delta_{S} / \nu<100$. Hence, by comparing the numerical and analytical solutions, both the NS model result accuracy and convergence rate can be assessed as a function of grid size and time step. 
(a)

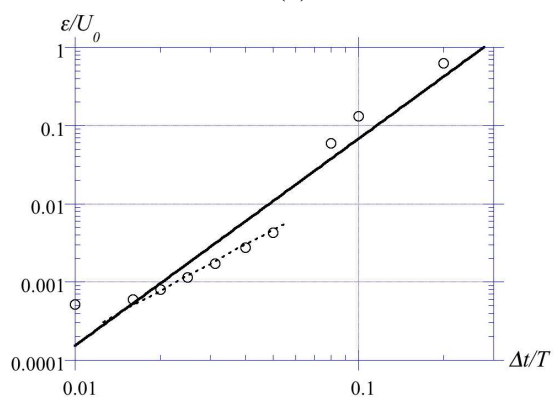

(b)

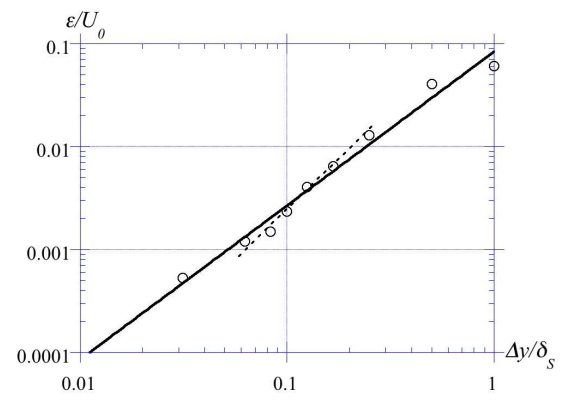

Figure 3: Relative error of computed velocity, as compared to Eq. 9, as a function of: (a) time step $\left(\Delta y=\delta_{S} / 64\right)$; (b) grid size $(\Delta t=T / 1000)$. Line (-) is a best power fit to the data, and (- - -) shows the 2 nd-order convergence.

To do so, we set $\mathbf{R e}=1, \delta_{S}=1, U_{o}=\pi$ and $\nu=\pi$. The NS domain dimensions are $h=16 \delta_{S}$ in each direction, and the spatial grids are identical in each direction (i.e., $\Delta x=\Delta y=\Delta z$ ). The model is ramped-up for $100 T$ and maximum relative errors on flow velocity $\varepsilon / U_{o}$, are calculated at $t=100 T$. Fig. 2 shows the analytic solution (Eq. 9) for 4 phase angles of the flow, $\phi=\omega t$ and Fig. 3 shows numerical errors when varying time step $\Delta t$ for a fixed grid size $\left(\delta_{S} / 64\right)$, or varying the grid size for a fixed time step $(\Delta t=T / 1000)$. Errors reduce as expected when reducing either grid size or time step and closely follow (or outperform) the 2nd-order convergence, expected from the model's discretization and time integration schemes, in the typical range of parameter values.

\section{Oscillatory Turbulent Boundary Layer}

This problem is similar in set-up and flow forcing to the previous one, but now for an oscillatory flow with higher Reynolds number over a rough flat plate. This creates a turbulent oscillatory boundary layer flow structure, for which there is no longer an analytical solution. In this case, model results are compared to Jensen et al.'s (1989) laboratory experiments, performed in an oscillating water tunnel (Utube), with flows driven by a pressure gradient. The bed roughness was $0.35 \mathrm{~mm}$ in experiments (sand paper). For this case as well, there is no need for running the NWT, but results nevertheless allow to assess the accuracy of all the important features of the NS-LES model, i.e., regarding flow forcing, bottom boundary condition, and turbulence representation. A new parallel implementation of the code is also being validated here.

The NS-LES model physical parameters are selected to match experiments (case 13) with $U_{0}=2 \mathrm{~m} / \mathrm{s}, T=9.72 \mathrm{~s}, \nu=1.1410^{-6} \mathrm{~m}^{2} / \mathrm{s}$ (i.e., $\delta_{S}=1.9$ $\mathrm{mm}$ and $\mathbf{R e}=3,295$ ), and $u_{*}=0.11 \mathrm{~m} / \mathrm{s}$ (as measured from the boundary layer structure near the bed). In the model, we specify a log-layer near the rough plate, with $z_{o}=k_{s} / 30=0.028 \mathrm{~mm}$, which yields $u_{*}=0.104 \mathrm{~m} / \mathrm{s}$ for the Smagorinsky scheme, thus in close match with experiments. The NS computational domain 
(a)

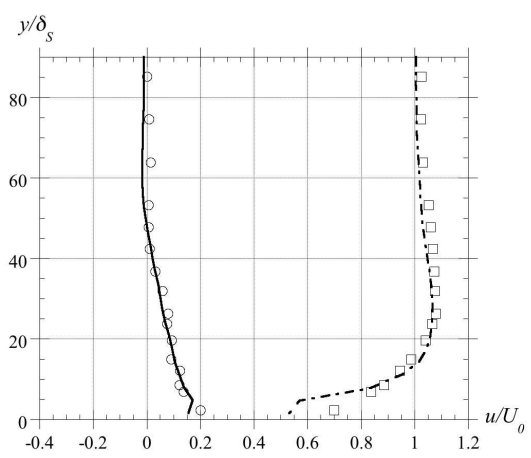

(b)

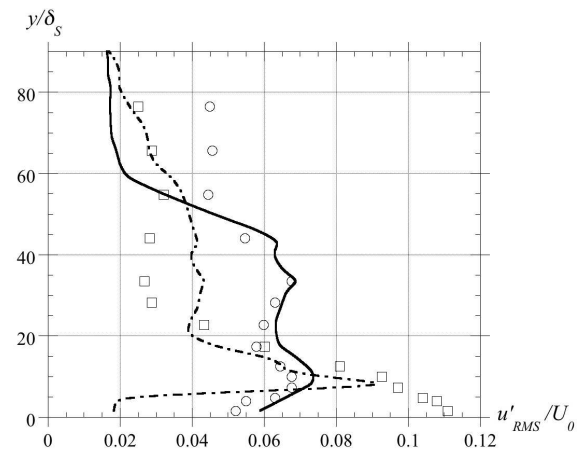

Figure 4: Horizontal velocity in NS-LES model (-), (- - -), vs. Jensen et al.'s (1989) experiments (symbols $\circ$ for $\phi=0$ deg.; $\square$ for $\phi=90$ deg.): (a) mean velocity; (b) RMS of turbulent velocity.

is $0.384 \mathrm{~m}$ long in the $y$ direction and half that in each lateral direction; spatial discretization is constant in each direction as $\Delta x=\Delta y=\Delta z=6 \mathrm{~mm}$ and time step is $\Delta t=0.001 \mathrm{~s}$. The NS-LES solution is ramped-up for $10 T=97.2 \mathrm{~s}$, and both mean and turbulent velocities are computed for $t=10 T$ and compared to experimental measurements.

Fig. 4a first shows that the mean horizontal velocity predicted in the model $\left(u=U=U^{I}+U^{P}\right)$ is in close agreement with experimental measurements at two phases of the flow. Fig. $4 \mathrm{~b}$ then shows that the RMS of the horizontal turbulent velocity $\left(u^{\prime}=u^{\prime} P\right)$ is in reasonable agreement with measurements at the same phases of the flow. This confirms the overall soundness of the NSLES model in representing turbulent oscillatory boundary layers, such as typically induced by ocean waves near the rough seabed. The good agreement of turbulent flow features, in particular, is key to the correct modeling of the suspension and transport of fine non-cohesive (i.e., sandy) sediment. This is illustrated in the next application of the full hybrid model.

\section{Sediment Transport Around Bottom Obstacle Due to Periodic Waves}

In this application, the set-up is similar to that shown in Fig. 1, and also detailed in (Grilli et al. 2003; Gilbert et al. 2007). Periodic FNPF waves are generated in the 2D-NWT and propagate over a small partly buried cylinder. The NS-LES model domain is a small box located around the obstacle and enclosing a small portion of the seabed with it.

Here, we present typical results of flow and sediment transport induced over a flat bottom, around a $75 \%$ buried circular cylinder of $8.4 \mathrm{~cm}$ radius, in a sandy seabed $\left(d_{50}=0.2 \mathrm{~mm}, \rho_{s}=2,650 \mathrm{~kg} / \mathrm{m}^{3}, w_{s}=0.026 \mathrm{~m} / \mathrm{s}, k_{s}=1 \mathrm{~mm}\right)$ by a (nonlinear) periodic wave of height $H=0.12 \mathrm{~m}$ and period $T=2.94 \mathrm{~s}$, in water depth $d=0.8 \mathrm{~m}$ (these are in fact laboratory scales of earlier experiments 
(a)

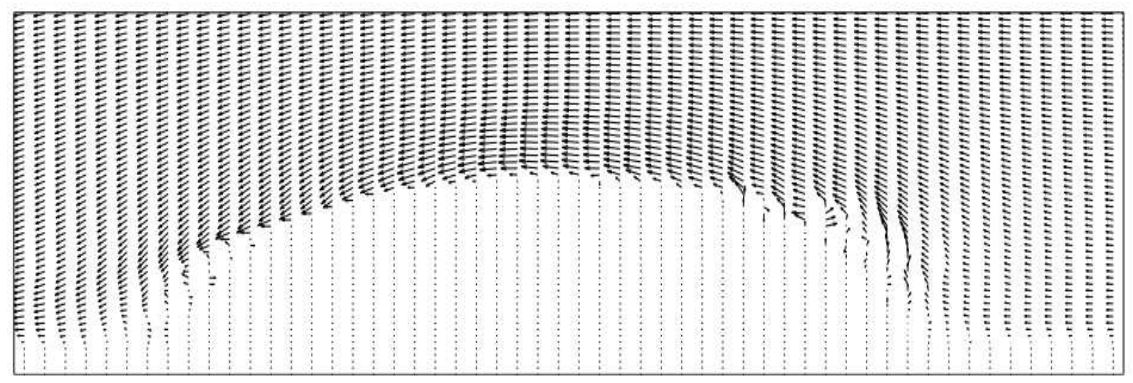

(b)

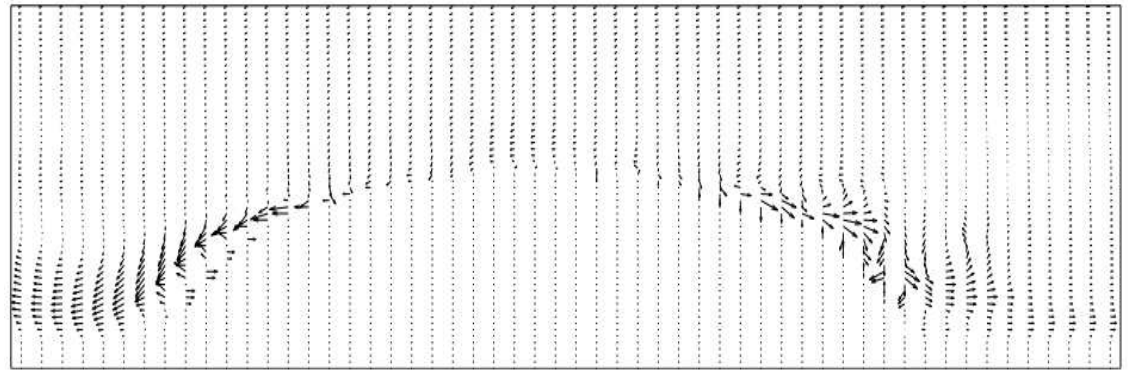

(c)

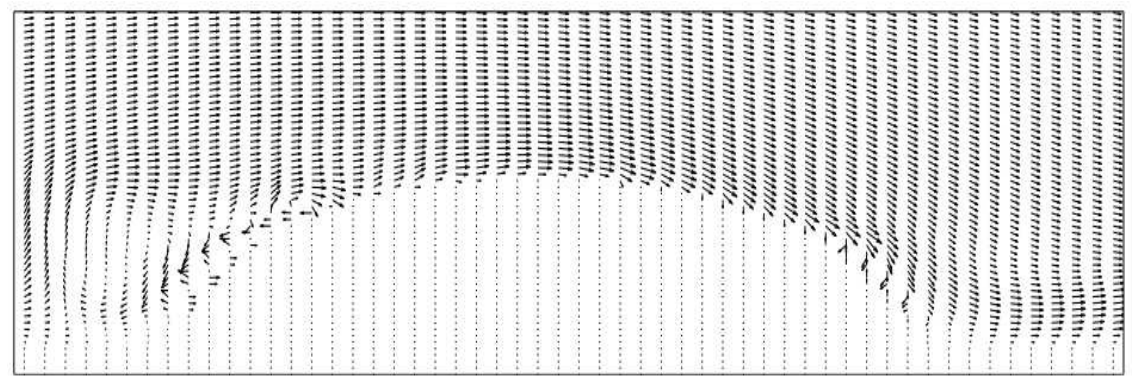

(d)

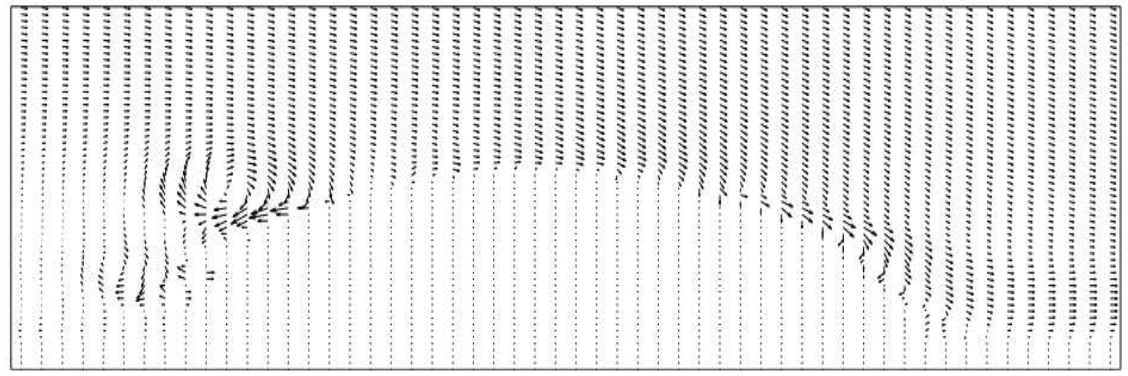

Figure 5: Wave-induced (mean) velocity fields $\left(y=0, U_{i}^{I}+U_{i}^{P}\right)$ in NS-LES model for periodic wave flow, forced around a $75 \%$ buried circular cylinder. (a)-(d) denote four stages of the flow during one wave period $T$. [Velocity varies from $-0.37-0.38 \mathrm{~m} / \mathrm{s}$.] 
(a)

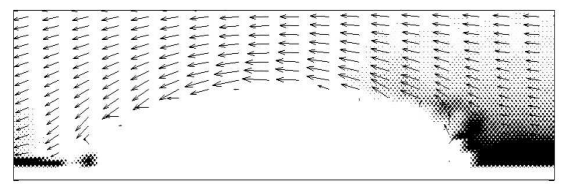

(c)

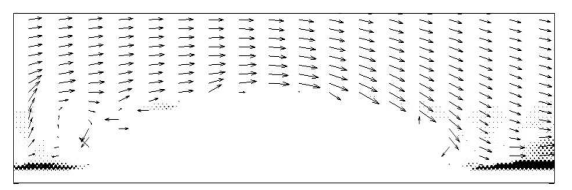

(b)

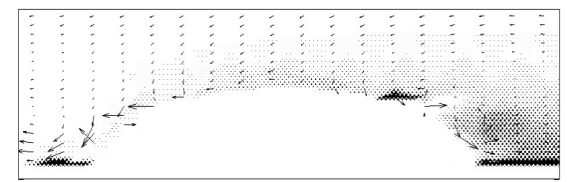

(d)

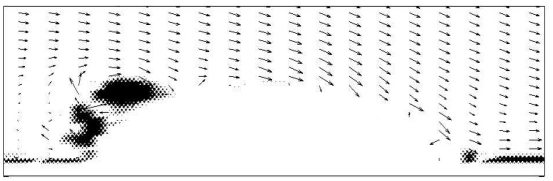

Figure 6: Wave-induced (mean) sediment concentration (as a gray scale) $(y=0, \bar{C})$ in NS-LES model ((a)-(d) are cases of Fig. 5). Vortices pickup and transport eddies of suspended sediment, reaching concentrations of up to $\overline{\mathrm{C}} \simeq 10^{-6}$.

performed with a moving sandy bed; Grilli et al. 2003).

Similar simulations were performed with the earlier version of the model (Gilbert et al., 2007), but faced numerical instabilities at the matching boundary of the two models, requiring sponge layers. Present computations with the new $P$-field implementation do not experience such instabilities.

NWT discretization. Typical free surface discretization (on $\Gamma_{f}$ ) in the 2D-NWT usually have at least 20 nodes per dominant wavelength. Lateral boundaries $\left(\Gamma_{w}\right.$ and $\Gamma_{p}$ ) are typically discretized with only 7-11 nodes. Bottom discretization (on $\left.\Gamma_{b}\right)$ are usually a little coarser than on the free surface, but horizontal node spacing $(\Delta x)$ is reduced on the bottom under the NS-LES domain, in order to increase the accuracy of integrals used to compute internal fields in the BEM solution. Initial time steps are selected based on the free surface node spacing, to satisfy the optimal mesh Courant number, and subsequently automatically adjusted. Typical CPU times in the NWT are less than one second per time step on a desktop computer.

Here, the NWT was run without bottom friction, and with no regridding applied on the free surface, because no long term computations were required for such simple periodic waves; in fact only a few (10) periods of wave forcing were used in the NS-LES model. An absorbing beach AB and an absorbing piston AP (Fig. 1) are specified in order to damp incident wave energy and eliminate (or reduce) wave reflection at the NWT extremity.

LES model discretization. The NS-LES model domain dimensions are $0.3 \mathrm{~m}$ long, by $0.173 \mathrm{~m}$ high, by $0.0125 \mathrm{~m}$ wide. For the partially buried obstacle, the bottom is flat in the streamwise direction to either side of a central bump, represented by a $75 \%$ buried cylinder of radius $0.084 \mathrm{~m}$, whose axis runs in the spanwise direction. The NS-LES model is discretized by $82 \times 98 \times 16$ grid points. Hence, $82 \times 98=8,036$ points are used in each vertical plane of the 3D NS-LES domain. 
The immersed boundary is located above the actual grid boundary so that the total flow domain height is $0.17 \mathrm{~m}$ (discretized by 95 points in the vertical direction over the flat regions). The obstacle protrudes a vertical distance of $0.042 \mathrm{~m}$ into the flow. The wave forcing is two dimensional, as is the bed and, based on our previous work (Gilbert et al. 2007), the main structure of vortices in the vertical plane is not significantly influenced by cross-stream resolution in channel flows. Accordingly, we used a smaller number of of cross-stream grid points, assuming that while the flow will not be as well resolved in that direction, the results in the vertical plane are not noticeably impacted. No change in the model or setup, however, is required to increase the cross-stream resolution, but the computer time required increases rapidly as one does that. [Such cases with finer lateral discretization will be run in the near future, with the newly developed MPI parallel version of the code, on large computer clusters.]

For the coupled 2D-NWT/3D-NS simulations with a periodic wave flow, wave forcing is computed in the NWT at the grid cell centers of the the fixed embedded NS-LES domain around the obstacle. Thus, the NWT provides the far field velocities and free-surface wave forcing throughout most of the domain $\Omega$, while the embedded NS solution provides a well-resolved description of the wave boundary layer within its domain, with no slip enforced at the bed. Wave generation is ramped-up over three periods in the NWT and computations are performed until a quasi-steady regime is reached. This usually takes 10 wave periods or so. Internal velocity fields are then calculated in the NWT to both initialize and subsequently force the NS-LES model. There is no NWT forcing in the transverse direction for this 2D flow, but turbulent fluctuations will carry momentum laterally to the main flow direction, in the NS-LES model.

Examples of velocity and sediment concentration results computed in the NSLES model around the cylinder are shown in Figs. 5 and 6, for the eleventh period of run time (the first 10 periods are model ramp-up). In this case, the suspended sediment concentration field was initialized as a function of the bottom shear stress at the end of the tenth period. Results are shown in a vertical plane at $y=0$. [Bedload was not computed for this initial illustration of our coupled model computations.]

Characteristic of all boundary layers, velocity profiles shown in Fig. 5 tend to zero along the bed, with larger near-bed velocities surrounding the crest of the cylinder. Shear layers form in the lee of the obstacle during wave phases where the velocity tapers off from its maximum value, as would be expected for a typical oscillatory flow case. As the flow slows enough so that it is about half its maximum value, some of the near-bed velocities reverse and form what looks to be the beginning of a typical lee vortex. However, as the flow slows to zero, the pressure gradient distribution on the cylinder from the NWT acts to intensify the flow in the direction down the slope. This jet of fluid is now moving in the direction opposite to the new flow direction. After the flow switches direction, this near-bed jet of fluid, which opposes the new main flow direction, rolls up into 


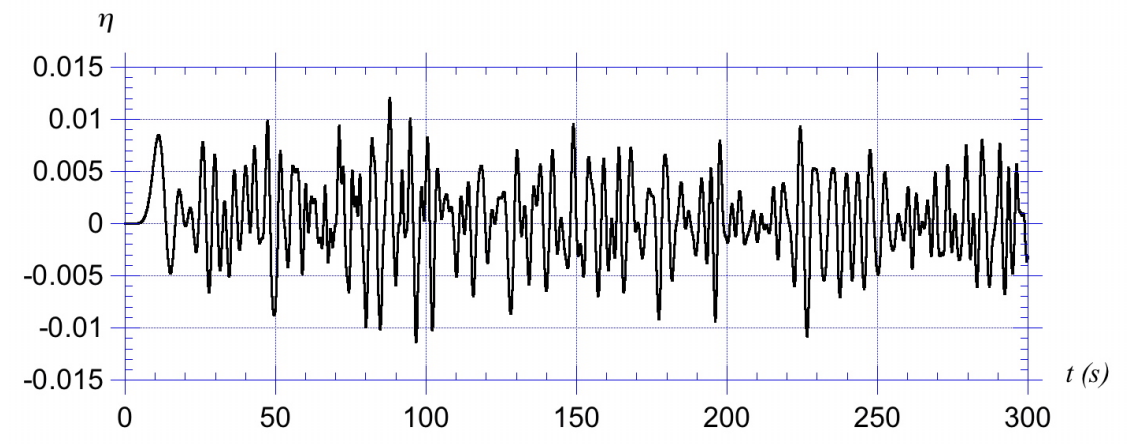

Figure 7: Typical irregular waves generated in the 2D-NWT based on a specified JONSWAP spectrum (Greene 2008).

a spanwise vortex on the stoss side of the cylinder.

As expected, the sediment transport patterns in Fig. 6 follow the flow field very closely. However, the entrainment patterns differ significantly because the boundary condition along the cylinder enforces the condition $C=0$. Strictly, this is only a first approximation to the correct boundary condition, which would allow for deposition and subsequent pickup on the obstacle boundary $\Gamma_{b}$. For the case studied here, this approximate boundary condition may have contributed to oscillations in the concentration profiles, which eventually lead to somewhat unrealistic behavior in the sediment concentration field. In general, sediment is picked up where the shear stresses are greater than critical along the flat bottom regions both upstream and downstream of the cylinder. It is then oscillated back and forth over the mine due to the action of the flow. More details and discussions of such flow and sediment patterns can be found in (Gilbert et al. 2007).

\section{SUMMARY AND PERSPECTIVES}

The validations and applications of hybrid model coupling methodologies presented here show that, by seamlessly combining and integrating the best features of different fluid models, with different (but relevant) "physics" (and/or spatial and temporal scales), in various parts of the fluid domain, one can achieve both an accurate and efficient solution of complex free surface flow problems, including in the presence of structures and with complex seabed dynamics.

While the presented applications were only aimed at both illustrating and validating model features, and hence were of limited complexity and size, the recent MPI parallel implementation of the models on very large computer clusters makes it possible simulating problems of increasingly practical interest and relevance to various fields of engineering. Such tools can also be used to investigate and gain insight into complex physical processes involving, e.g., turbulent boundary layer flows, the interaction of those with rigid structures, and/or a moving bed, and the sediment transport that might result. 
While the present paper was devoted to the coupling of FNPF-NWT and a (submerged) NS-LES model, which have been spearheaded by Grilli and coworkers, the same hybrid approach can of course be (and is being) used to couple other types of models, such as FNPF/HOS (Higher-order Spectral) or NWT models with NS models around surface piercing fixed or floating structures (e.g., Alessandrini 2007), or long wave model with NS-VOF or SPH (Smoothed Particle Hydrodynamics) models; this currently being researched by various other groups.

Although this aspect has not been used here, the 2D-NWT was recently improved (Greene 2008), both in efficiency for large discretization (through using a localized Green's function leading to sparse matrices, and a MPI parallel implementation) and in its generation of well-controlled irregular wave sea states, e.g., such as generated using a flap wavemaker motion based on a specified JONSWAP energy spectrum. A few iterations are performed in the generation, until the target spectrum is satisfactorily created in the NWT (e.g., Fig. 7). Once the development and implementation of the full hybrid model are complete, including the possibility of running the model on very large computer clusters, such irregular wave forcing will be used to perform more realistic long term simulations of wave-induced sediment transport and resulting scouring or burial of a bottom obstacle. The latter aspect of moving bed is still being developed and validated.

\section{ACKNOWLEDGEMENTS}

The authors gratefully acknowledge support from the US Office of Naval research (code 321CG), under Grants: N00014-00-1-0440 and N00014-05-1-0068.

\section{REFERENCES}

Alessandrini, B. (2007). Thèse d'Habilitation en Vue de Diriger les Recherches. Ecole Centrale de Nantes, Nantes, $\mathrm{PhD}$ thesis.

Biausser B., S.T. Grilli, Fraunie P. and Marcer, R. (2004) Numerical analysis of the internal kinematics and dynamics of three-dimensional breaking waves on slopes. Intl. J. Offshore and Polar Engng., 14(4), 247-256.

Corte, C. and Grilli S.T. (2006). Numerical Modeling of Extreme Wave Slamming on Cylindrical Offshore Support Structures. Proc. 16th Offshore Polar Engng. Conf., 3, 394-401.

Cui, A. and R.L. Street (2001). Large-eddy simulation of turbulent rotating convective flow development, J. Fluid Mech., 447, 53-84.

Dommermuth, D. G., Yue, D. K. P., Lin, W. M., Rapp, R. J., Chan, E. S. and Melville, W. K. (1988). Deep-water plunging breakers: a comparison between potential theory and experiments. J. Fluid Mech., 189, 423-442.

Fochesato C., Grilli, S.T. and Dias F. (2007). Numerical modeling of extreme rogue waves generated by directional energy focusing. Wave Motion, 44, 395-416.

Greene, N.A. (2008). Irregular wave generation in a fully nonlinear potential flow numerical wave tank, Masters Thesis, University of Rhode Island. 
Gilbert R.W., Zedler E.A., Grilli S.T., and Street R.L. (2007). Progress on NonlinearWave-Forced Sediment Transport Simulation. IEEE J. Oceanic Engng., 32(1), 236-248.

Grilli, S.T. (1997). Fully Nonlinear Potential Flow Models used for Long Wave Runup Prediction. Chapter in Long-Wave Runup Models, (eds. H. Yeh, P. Liu, and C. Synolakis), pps. 116-180. World Scientific Publications, Singapore.

Grilli, S.T. and Horrillo, J. (1997). Numerical Generation and Absorption of Fully Nonlinear Periodic Waves. J. Engng. Mech., 123(10), 1060-1069.

Grilli, S.T. and Subramanya, R. (1996). Numerical Modeling of Wave Breaking Induced by Fixed or Moving Boundaries. Comput. Mech., 17, 374-391.

Grilli, S.T., Voropayev, S., Testik, F.Y. and Fernando, H.J.S. (2003). Numerical Modeling and Experiments of Wave Shoaling Buried Cylinders in Sandy Bottom. Proc. 13th Offsh. and Polar Engng. Conf., 3, 405-.

Helluy Ph., Golay F., Caltagirone J.-P., Lubin P., Vincent S., Drevard D., Marcer R., Seguin N., Grilli S., Lesage A.-C. and Dervieux A. (2005). Numerical simulations of wave breaking. Math. Modeling and Numer. Analys., 39(3), 591-607.

Jensen, B. L. , B. M. Sumer, and J. Fredsoe (1989). Turbulent oscillatory boundary layers at high Reynolds numbers. J. Fluid Mech., 206, 265-297.

Leonard, B. P. (1979). A stable and accurate convective modelling procedure based on quadratic upstream interpolation. Comput. Meth. in Applied Mechanics and Engng., 19, 59-98.

Leonard, B. P. (1988). Simple high accuracy resolution program for convective modeling of discontinuities.Intl. J. Numer. Meth. Fluids, 8, 1291-1318.

Lin, P. and Liu, P. L.-F. (1998). A numerical study of breaking waves in the surf zone. J. Fluid Mech., 359, 239-264.

Lubin, P. (2004). Large Eddy Simulations of plunging breaking waves. Ph.D Dissertation, University of Bordeaux I, France.

New, A. L., McIver, P. and Peregrine, D. H. (1985). Computations of overturning waves. J. Fluid Mech., 150, 233-251.

Zang, Y., Street, R. L. and Koseff, J. R. (1994). A non-staggered grid, fractional step method for time-dependent incompressible Navier-Stokes equations in curvilinear coordinates J. Comput. Phys., 114, 18-33. 